\title{
Evaluating Effect of Anxiety on Eye Hand Coordination Using Jenga Game in Female Nursing Professionals: An Observational Study
}

\author{
Dr. Jeba Chitra ${ }^{1}$ \\ Professor, Neurophysiotherapy \\ Department \\ KAHER Institute of Physiotherapy \\ Belagavi, India.
}

\author{
Dr. Jorida Fernandes ${ }^{2}$ \\ Lecturer, Neurophysiotherapy \\ Department \\ KAHER Institute of Physiotherapy \\ Belagavi, India.
}

\author{
Jowena Parikh ${ }^{3}$ \\ Post-graduate student, \\ Neurophysiotherapy Department \\ KAHER Institute of Physiotherapy \\ Belagavi, India
}

\begin{abstract}
Amongst the general working population, female nurses were observed to have higher levels of anxiety and performed poorly under the influence of anxiety. It is still a dispute as to how it affects perceptual motor performance. Thus, this study evaluated correlation between Eye Hand Coordination and Anxiety in 67 female nursing professionals using Jenga game and Hamilton Anxiety Rating scale (HAMA). A negative correlation was perceived. This study concluded that anxiety affected motor performance. Future scope of this study would involve working on methods to cope with anxiety and improve Eye hand Coordination skills which would help nurses work efficiently.
\end{abstract}

Keywords:- Anxiety; Eye Hand Coordination; Jenga; Nursing Professionals.

\section{INTRODUCTION}

Emotions and anxiety predominate everyday lives. The study of emotion, principally its impact on various traits of behavior have greeted much attention in literature. ${ }^{1}$ As per surveys conducted in large populations, 33.7 percent of the population is affected by anxiety during their lifetime. ${ }^{2}$ Physiological symptoms of anxiety can include trembling, shortness of breath, chest pain, dizziness, nausea, blushing or sweating. ${ }^{3}$ In comparison to men, women are significantly more likely to develop an anxiety disorder over their lifespan. ${ }^{4}$ Amongst the general population, nurses were found to have higher levels of anxiety, as per a study conducted in China in the year 2015. 5 Therefore it can be said that a commitment to provide quality health care can be impaired by chronic stress and can cause problems like ability to concentrate. ${ }^{6}$ Under anxiety an individual toils to perform a task and an increased dependency in daily activities could be attributed to a decline in Eye Hand Co-ordination (EHC). ${ }^{1,7}$

Eye-Hand Co-ordination (EHC) refers to the ability to produce a goal-oriented hand action which is guided by visual information from the eyes. ${ }^{8}$ Studies in cognitive neuroscience advocate that the time and location of docking the hand and eye on the object should be synchronized, for efficiently performing a goal-oriented task. Eye movements play a key role in carrying out a particular motor action, such that the feedback can be turned into a meaningful instruction. ${ }^{9}$ For this particular reason assessment of EHC plays an important role in understanding the functional processes that underlie goal-oriented behaviors such as reaching, grasping or positioning of the objects. Assessments of EHC included tests like Moore's Hand eye coordination test, Purdue Peg board test, Grooved pegboard test. ${ }^{7}$ Recent advances include playing Nintendo Wii games based on sports or cooking and other computer-based gaming. Certain games like Legos, Jenga and connect four are also considered to improve motor skills. ${ }^{10}$ Advantages of these games are that they are convenient, played by all age groups and easy to administer.

Jenga was developed by a game designer, Leslie Scott. The game requires the players to pile all the wooden blocks to form a tower. Once the tower is formed the participants are required to take one block from the tower and place it on the top, ensuring that the tower doesn't collapse. Plenty studies in neuropsychology have used this game for the purpose of assessing behavioral characteristics in children along with their communication skills and assessed co-ordination between parent and a child. ${ }^{11}$ However, it is uncommon in studies to consider the aspect of Eye Hand Co-ordination using this game. Although dissimilarities between attentional, interpretational and behavioral levels at which anxiety influences is vindicated, more work is needed to appropriately assess their respective impacts on a person's performance. Thus, the study presented here trials for the effect of anxiety on the Eye Hand Co-ordination using Jenga game.

\section{METHODOLOGY}

The present study involved analyzing the data from a cross-sectional study for an association between anxiety and Eye Hand Coordination, where 67 nurses within the age group of 25-45 years with normal or corrected to normal vision and a score of more than 13 on Hamilton Anxiety Rating scale (HAM-A) were included. Participants were recruited from KLES Dr. Prabhakar Kore Charitable Hospital and Medical Research Centre from October 2018 to March 2019. The study was approved by the ethical committee of the Institution. Participants with a recent history of trauma, eye surgeries, any neurological conditions, cognitive impairment and severe neuropsychological disorders were excluded. Subjects were screened on the basis of inclusion and exclusion criteria 
with explanation of the purpose and procedure of the study to the ones eligible. A written informed consent was obtained for the same and their demographic data was noted.

Assessment of Anxiety was done by using a scale called Hamilton Rating Scale (HAM-A), main purpose of which was to assess the severity of symptoms of anxiety. Scale consists of 14 items, each defined by a series of symptoms, both psychic (mental agitation and psychological distress) and somatic anxiety (physical complains related to anxiety). Each item is scored on a scale of 0 (not present) to 4 (severe), with a total range being 0-56, where below 17 indicates mild severity, 18-24 indicates mild to moderate severity and 20-30 moderate to severe. $^{12}$

The game of Jenga (TOMY Co. Ltd.), composed of 54 hardwood blocks, where a player removes wooden blocks from a stacked tower formation and place it on top of the tower which will make it less stable was used to assess EHC. Goal of the game is to keep the tower standing without falling. A player loses if they cause the block or tower to fall and are unable to remove a block on their turn. The scoring of EHC depended on the number of blocks the participant could place on top of the tower without making it fall.

HAM-A was handed out to the participants and a game of Jenga was played between the examiner and the subject with a time limit of 15 minutes. Based on the scores obtained, subjects were assessed for co-relation between Eye Hand Coordination and Anxiety.

All the statistical analysis was completed using the Statistical Package for Social Sciences (SPSS) software version 21. Descriptive data was found to follow a normal distribution thus parametric tests were applied. Normality of the distribution was calculated using the Kolmogorov Smirnov test. Karl's Pearson's correlation coefficient method was used to analyze the correlation between Anxiety and EHC scores, also between age and BMI to Anxiety and EHC score. An association between different age groups and levels of anxiety was calculated using Chi Square tests. One-way Analysis of variance (ANOVA) was done to obtain an age group-wise comparison with mean Anxiety and EHC scores. A pair wise comparison of age amongst the same was achieved using Tukey's multiple Post-hoc procedures. (See Figure 1)

\section{RESULTS}

The mean age of the total participants was 28.70 years \pm 3.68 , mean BMI score was $21.25 \pm 3.820$, mean anxiety score was $19.075 \pm 4.778$ and mean co-ordination scores obtained were $10.149 \pm 3.767$. There was a significant difference in the correlation between anxiety and Eye Hand Coordination scores. ( $\mathrm{p}<$ 0.05) [Table 1]. A negative correlation was obtained between anxiety and Eye Hand Coordination scores as seen in Figure 2. Subjects were evaluated for their levels of anxiety and their association with Eye Hand Coordination scores. An appreciable drop in the scores of Eye Hand Coordination was obtained with increase in the level of anxiety as seen in Table 2.

Different age groups were compared with their mean Anxiety and EHC scores, along with a comparison amongst the age groups for the same. Mean of the age groups showed no significance( $p>0.05)$ for Anxiety and EHC score. When a comparison of age groups in pairs amongst themselves was gained, no significant difference was seen in the age groups pair wise comparison. $(p>0.05)$ [Table 3 and Figure 3].

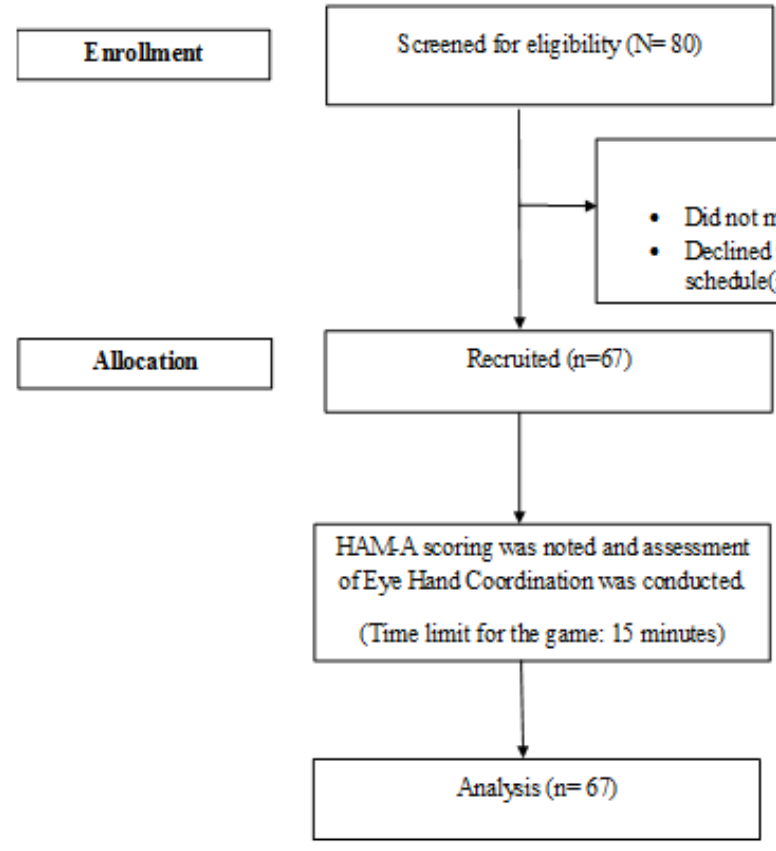

Fig 1:- Consort Diagram 
ISSN No:-2456-2165

\begin{tabular}{|c|c|c|c|}
\hline \multirow{2}{*}{ Variables } & \multicolumn{3}{|c|}{ Correlation of anxiety with EHC scores } \\
\cline { 2 - 4 } & r-value & t-value & p-value \\
\hline Coordination scores & -0.5546 & -5.3731 & $0.0001 *$ \\
\hline
\end{tabular}

Table 1:- Correlation between anxiety and Eye Hand Coordination scores.

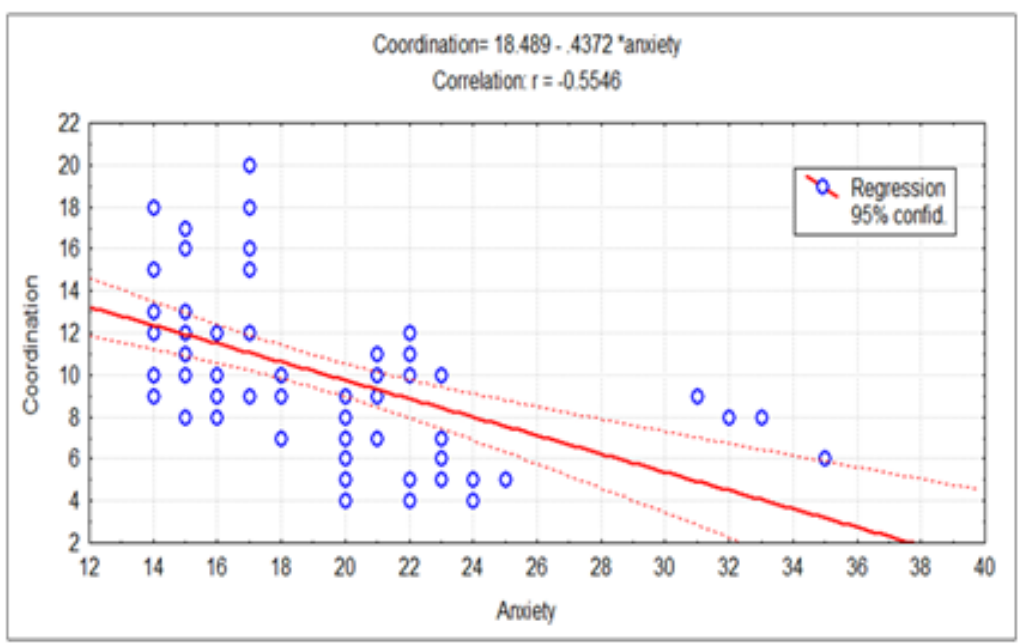

Fig 2:- Correlation between anxiety and Eye Hand Coordination scores.

\begin{tabular}{|c|c|c|c|}
\hline Level of anxiety & No. of Subjects & Mean EHC & SD EHC \\
\hline Mild anxiety & 33 & 12.79 & 3.14 \\
\hline Moderate anxiety & 29 & 7.66 & 2.36 \\
\hline Severe anxiety & 5 & 7.20 & 1.64 \\
\hline Total & 67 & 10.15 & 3.77 \\
\hline F-value & \multicolumn{2}{|c|}{$0.0001^{*}$} \\
\hline P-value & $\mathrm{P}=0.0001^{*}$ \\
\hline \multicolumn{2}{|c|}{$\mathrm{P}=0.0003^{*}$} \\
\hline Pair wise comparisons by Tukeys multiple posthoc procedures \\
\hline Mild anxiety vs Severe anxiety & $\mathrm{P}=0.9763$ \\
\hline
\end{tabular}

Table 2:- Level of anxiety and their correlation with EHC scores.

\begin{tabular}{|c|c|c|c|c|}
\hline \multirow{2}{*}{ Age groups } & \multicolumn{2}{|c|}{ Anxiety } & \multicolumn{2}{|c|}{ Coordination } \\
\hline & Mean & $S D$ & Mean & $S D$ \\
\hline $25-29$ & 18.156 & 3.890 & 10.311 & 3.698 \\
\hline $30-34$ & 20.778 & 5.579 & 10.278 & 4.184 \\
\hline $35+$ & 21.750 & 8.016 & 7.750 & 2.062 \\
\hline Total & 19.075 & 4.778 & 10.149 & 3.767 \\
\hline$F$-value & \multicolumn{2}{|c|}{2.7406} & \multicolumn{2}{|c|}{0.8597} \\
\hline p-value & \multicolumn{2}{|c|}{0.0721} & \multicolumn{2}{|c|}{0.4281} \\
\hline \multicolumn{5}{|c|}{ Pair wise comparisons by Tukeys multiple posthoc procedures } \\
\hline $25-29$ vs $30-34$ & \multicolumn{2}{|c|}{0.1159} & \multicolumn{2}{|c|}{0.9995} \\
\hline $25-29$ vs $35+$ & \multicolumn{2}{|c|}{0.3076} & \multicolumn{2}{|c|}{0.4003} \\
\hline $30-34$ vs $35+$ & \multicolumn{2}{|c|}{0.9245} & \multicolumn{2}{|c|}{0.4509} \\
\hline
\end{tabular}

Table 3:- Comparison of age groups with mean anxiety and EHC scores. 


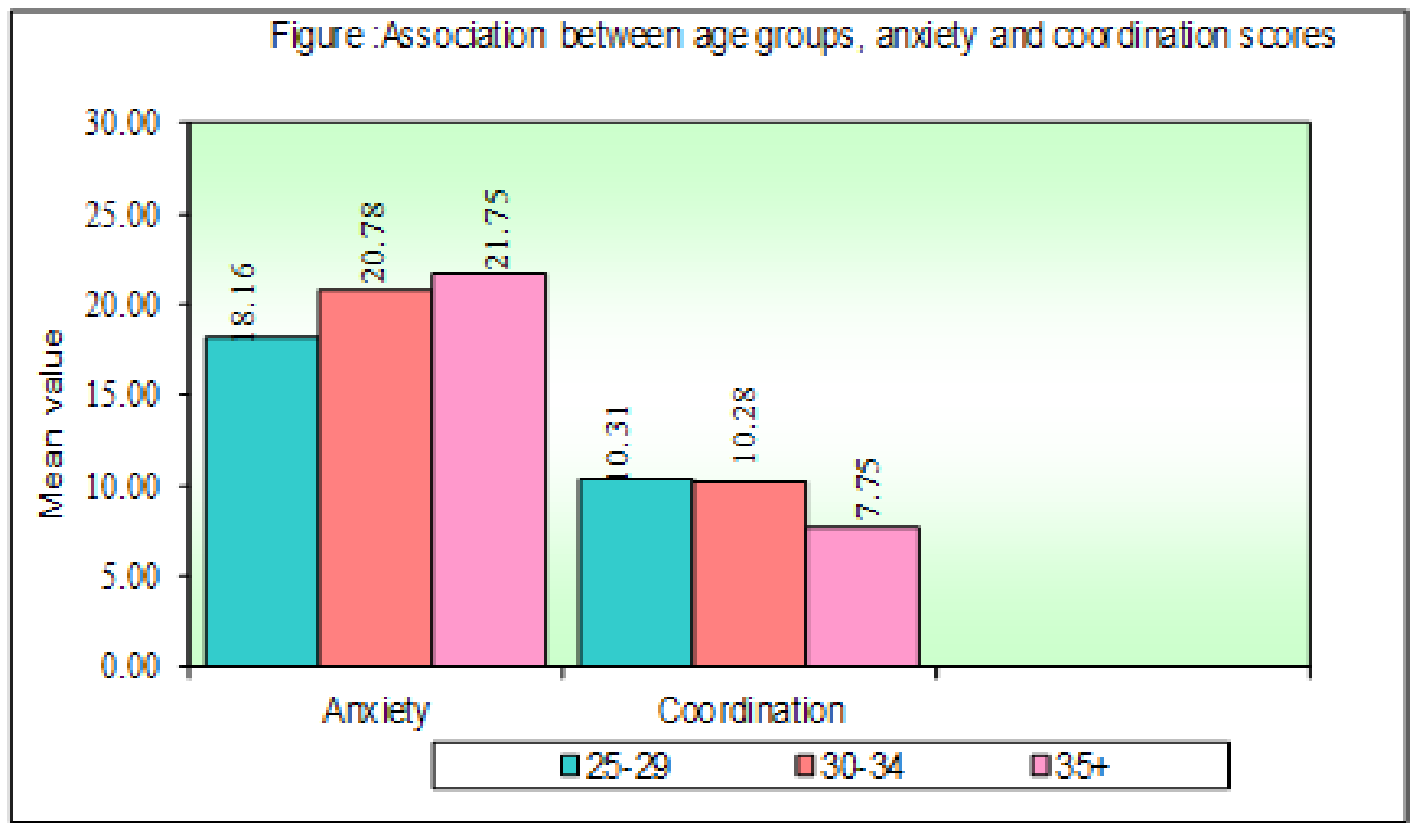

Fig 3:- Comparison of age groups with mean anxiety and EHC scores.

\section{DISCUSSION}

Rationale of this study was to analyze the correlation between Anxiety and Eye Hand Coordination using Jenga game and associating it to the level of anxiety, obtained on a HAM-A scale. Being one of the first scales developed, it is widely used in clinal trials, which assess both psychic and somatic anxiety. It is also a well validated tool, which assesses severity of anxiety. ${ }^{12}$ Other scales like, Becks Anxiety Inventory (BAI) and State-Trait Anxiety Inventory (STAI), used to evaluate anxiety do not specifically quantify the levels of anxiety, thus HAM-A was used. For earlier assessment of EHC tests like Grooved Peg board test, Purdue pegboard tests were used along with use of Nintendo Wii games for the same. Games like connect four, Legos, Jenga are known to improve certain motor functions and are easy to administer and hence convenient. However, there is a dearth of literature on as to how Jenga evaluates EHC, consequently the proposed study to evaluate the same.

Attention is a chief necessity when it comes to performing a task involving Eye Hand Coordination. ${ }^{13}$ Anxiety, however makes it challenging for an individual to complete a task with utmost efficiency due to the fact that with higher levels of anxiety, individual focuses more towards the threat or situation (attentional bias) rather than the task performed. An increased activation of amygdala in anxiety paired with decreased prefrontal control makes it difficult to perform a task. ${ }^{1}$ It could be hypothesized that the negative correlation obtained in the study could be due to the aforementioned reasons.

Another supposition to this correlation could also be owed to the influence of anxiety on gaze stability. ${ }^{14}$ There is a marked effect of reduction in gaze stability on visual targets under anxiety. ${ }^{7}$ The amount of time one spends on looking at the target is known to be linked to the goal- directed action performed. It could be theorized that anxiety related gaze diversion from task performance could have attenuated EHC score in the present study. Thus, with an increase in levels of anxiety, coordination scores evaluated could have reduced. Study results also corelate with a review conducted by Arne et al. (2012) on as to how anxiety affects perceptuo-motor performance, both positively and negatively. They stated that when it comes to movement execution, an individual must generate coordination patterns and muscle activity to make their actions possible and that this goal directed action may be impaired due to anxiety. Thus, it could be conceptualized that under anxiety, there is an increase in corticospinal motor tract excitability which activates the muscle causing more force production therefore indirectly impacting how the movements are performed. ${ }^{1}$

Depending on the levels of anxiety, whether mild, moderate or severe, a consistent drop in the mean EHC scores was seen. Also, a pair wise comparison between three different levels of anxiety when done, stated a significant difference between the scores obtained on mild versus moderate and mild versus severe anxiety levels. However, no significant differences were obtained between mean EHC scores for moderate versus severe anxiety, which may have been due to a lesser number of people being reported with severe anxiety. Previous studies focus on explaining this anxiety performance relationship using a theory, namely, the Attentional Control theory which speculates that worry diminishes a person's ability to oppose interference from a task irrelevant stimulus. This, indirectly causes an imbalance in the goal directed attentional systems, consequently reducing efficiency of the task to be performed. ${ }^{15}$ Applicability of this theory to the present study may explain the decrement in the performance scores with hiked levels of anxiety. It can thus be stated that anxiety sways the performance behavior by impacting perception and working memory. 
Execution of a task under fretfulness may also brunt an individual's self-consciousness about performing well, persuading them in a conscious thought process during the performance, leading to an eventual disruption in the accomplishment of a task. The disseminated neuronal connections within the right prefrontal cortex (PFC) manifests a crucial role in anxious arousal. Therefore, with inflation in anxiety, the PFC becomes unshielded towards disruption that results due to it, making it no longer available to keep up with the ongoing task. ${ }^{16}$ This interdependence between anxiety and cognition was also explained by Katherine Vytal et. al (2013), wherein they explained an occurrence of disruption in the spatial and working memory performance on induction of anxiety. The subjects in the study underwent a series of spatial and verbal $n$-back tasks at a varied difficulty level while they were safe or at a risk of shock. A negative correlation between these parameters was obtained, the results of which are consistent with the present study. ${ }^{17}$

Age group wise comparison of anxiety and EHC scores was done for understanding association between the same, if any. Within different age groups, it was observed that with an increasing age there was a surge in anxiety. Similarly, reduction in motor performances associated with coordination were seen with an increasing age. Nevertheless, significance of these parameters was seen clinically but not statistically. This may be explained due to the hormonal imbalances occurring with increased age, increased work-related stress or pregnancy. Reduction noted in coordination scores with ascending age could be due to increased anxiety levels, probable changes in visual perception, associated stress or depression or other physiological symptoms.

\section{CONCLUSION}

Therefore, this study concludes that with a raised level of anxiety, motor performance involving $\mathrm{EHC}$ is diminished. Clinical implication of this study models the basis for the fact that nurses should be trained not only on a professional front but also proficiently trained to deal with the psychological aspect. This will indirectly help them execute their tasks more efficiently, attributing to their improved EHC Skills. Limitations of the study involve the fact that HAM-A scale poorly discriminates between anxiolytic and antidepressant effects, and somatic anxiety versus somatic side effects. Also, generalizability of the study is limited, since only evaluates the nursing professionals.

\section{FUTURE SCOPE}

Future scope of this study should possibly be fixated on training their EHC skills. This could be achieved by various methods, including task stimulation procedures, where they are trained to work in a simulated stressful environment. Furthermore, studies to asses individual's coping with anxiety or reduction in anxiety post EHC skill training could be conducted. Also, Eye hand Coordination skills could be improved by using virtual reality methods, games like Jenga, Connect Four to name a few. Ultimately, this study could establish footing for new approaches to deal with anxiety and its consequences on motor performances mainly considering Eye hand Coordination skills.

\section{REFERENCES}

[1]. Arne Nieuwenhuys, Raôul R. D. Oudejans (2012). Anxiety And Perceptual-Motor Performance: Toward An Integrated Model Of Concepts, Mechanisms, And Processes. Psychological Research 76:747-759.

[2]. Borwin Bandelow,Sophie Michaells (2015). Epidemiology Of Anxiety Disorders In The $21^{\text {st }}$ Century. Dialogues In Clinical Neuroscience17:327335.

[3]. Erin L. Cassidy, Pamela J. Swales, Javaid I. Sheikh(2002) . Clinical Features of Anxiety Disorders. Principles and Practice of Geriatric Psychiatry ;2:551-554.

[4]. Pinal A. Patel, Prerna P. Patel, Anuradha V. Khadilkar, S. A. Chiplonkar, Ashish D. Patel(2016). Impact of Occupation on Stress and Anxiety Amongst Indian Women. Women \& Health.

[5]. Teris Cheung, Paul S.F(2015). Yip. Depression, Anxiety and Symptoms of Stress among Hong Kong Nurses: A Cross-sectional Study. Int. J. Environ. Res. Public Health 12, 11072-11100.

[6]. Alan P. Wolfgang(1988). Job Stress in the Health Professions: A Study of Physicians, Nurses, and Pharmacists. Hospital Topics; 66:4, 24-27.

[7]. Shirley S. M. Fong, Shamay S. M. Ng, Yoyo T. Y. Cheng, Janet Y. H. Wong, Esther Y. T. Yu, Gary C. C. Chow, Yvonne T. C. Chak, Ivy K. Y. Chan, Joni Zhang, Duncan Macfarlane, LouisaM. Y. Chung(2016). Effects of Ving Tsun Chinese Martial Art Training on Upper Extremity Muscle Strength and Eye-Hand Coordination in Community-Dwelling Middle-Aged and Older Adults: A Pilot Study. Evidence-Based Complementary and Alternative Medicine:1-7.

[8]. Kiseok Leea, Barbara M. Junghansa, Malcolm Ryanb, Sieu Khuua, Catherine M. Suttlea(2014). Development of a Novel Approach to the Assessment of Eye-Hand Coordination. Journal of Neuroscience Methods:50-56.

[9]. Mark Wilson, Mark Coleman, John McGrath(2010). Developing Basic Hand-Eye Coordination Skills For Laparoscopic Surgery Using Gaze Training. BJU International:1356-1358.

[10]. Sara Taft (2014). Hand Function Evaluation for Dental Hygiene Students. Electronic Theses and Dissertations:1-86.

[11]. Jenga.com [Internet]. Fun Facts : Jenga, edge of your seat fun. [2016]. Available from : http//jenga.com/.

[12]. Hamilton, M. The assessment of anxiety states by rating. British Journal of Medical Psychology 32:50$55,1959$. 
[13]. Chris Englert and Alex Bertrams (2012). Too Exhausted for Operation? Anxiety, Depleted Selfcontrol Strength, and Perceptual-motor Performance. Self and Identity, 1-13.

[14]. Jeffery P. Staab (2014). The Influence Of Anxiety On Ocular Motor Control And Gaze. Curr Opin Neurol,27:118-124.

[15]. Howie J. Carson \& Dave Collins (2016). The fourth dimension: A motoric perspective on the anxietyperformance relationship, International Review of Sport and Exercise Psychology, 9:1, 1-21.

[16]. Alexander J. Shackman, Jeffrey Maxwell (2006). Anxiety selectively disrupts visuospatial working memory. Emotion; 6:40-61.

[17]. Katherine E. Vytal, Brian R. Cornwell, Allison M. Letkiewicz, Nicole E. Arkin and Christian Grillon (2013). The complex interaction between anxiety and cognition: insight from spatial and verbal working memory. Frontiers in Human Neurosciences, 7 : 1-11. 